\title{
Range Performance Evaluation from the Flight Tests of a Passive Electro-Optical Aircraft Detection Sensor for Unmanned Aircraft Systems
}

\begin{tabular}{|r|l|}
\hline Journal: & Journal of Unmanned Vehicle Systems \\
\hline Manuscript ID & juvs-2014-0022.R2 \\
\hline Manuscript Type: & Article \\
\hline Date Submitted by the Author: & $19-$-Jan-2016 \\
\hline Complete List of Authors: & $\begin{array}{l}\text { Minwalla, Cyrus; National Research Council Canada, Aerospace Portfolio - } \\
\text { Flight Research } \\
\text { Thomas, Paul; York University, Lassonde School of Engineering - Vision } \\
\text { Sensor Laboratory } \\
\text { Ellis, Kristopher; National Research Council Canada, Aerospace Portfolio - } \\
\text { Flight Research } \\
\text { Hornsey, Richard; York University, Lassonde School of Engineering - Vision } \\
\text { Sensor Laboratory } \\
\text { Jennings, Sion; National Research Council Canada, Aerospace Portfolio - } \\
\text { Flight Research }\end{array}$ \\
\hline Keyword: & $\begin{array}{l}\text { Sense-and-avoid, unmanned aircraft systems, passive optical, range } \\
\text { performance, flight test }\end{array}$ \\
\hline
\end{tabular}




\title{
Range Performance Evaluation from the Flight Tests of a Passive Electro-Optical Aircraft Detection Sensor for Unmanned Aircraft Systems
}

\author{
Cyrus Minwalla ${ }^{a}$, Paul Thomas ${ }^{b}$, Kristopher Ellis $^{a}$, Richard Hornsey $^{b}$, Sion Jennings $^{a}$ \\ ${ }^{a}$ Flight Research Laboratory - Aerospace Portfolio, NRC Canada, Ottawa, Canada \\ ${ }^{b}$ Vision Sensor (VISOR) Laboratory, York University, Toronto, Canada
}

\begin{abstract}
The range performance evaluation of a multi-camera electro-optical aircraft detection instrument, "DragonflEYE", was conducted. A "range at first detection" $\left(R_{0}\right)$ quantity, evaluated from the temporal signal-to-noise ratio of potential targets on a collision course, is proposed as a generic metric for evaluating electro-optical systems. The methodology and evaluation process are discussed. Efficacy of the approach was tested by flying multiple collision trajectories, with the instrument mounted onto a Bell 205 helicopter acting as a surrogate unmanned aircraft system, while an instrumented Bell 206 Jet-Ranger acted as the intruder. The $R_{0}$ values were extracted and subsequently compared to visual estimates by the flight crew. A mean detection range of $R_{0}=6.3 \pm 1.7 \mathrm{~km}$ was observed to be within the margin of error for flight-crew detection range of $4.8 \pm 2.0 \mathrm{~km}$. Sensitivity analysis was conducted on the choice of threshold and the sensor's angular resolution, with increased resolution yielded diminishing returns due to atmospheric extinction. Robustness was assessed by repeating the experiment on a different day with a secondary camera array, "Cerberus", recording images simultaneously. The observed detection ranges were within the margin of error of prior estimates. In addition, measured ranges from Cerberus aligned with their predicted values.
\end{abstract}

Keywords: Sense-And-Avoid, electro-optical, passive, unmanned aircraft systems, range performance, flight test

\section{ABBREVIATIONS}

$\begin{array}{ll}\text { ADS-B } & \text { Automatic Dependent Surveillance - Broadcast } \\ \text { AGL } & \text { Above Ground Level } \\ \text { AILS } & \text { Airborne Information for Lateral Spacing } \\ \text { COTS } & \text { Commercial Off-The-Shelf } \\ \text { DN (dn) } & \text { Digital Number } \\ \text { EDSC } & \text { Embedded Distributed Smart Cameras } \\ \text { EO } & \text { Electro-Optical } \\ \text { EST } & \text { Eastern Standard Time } \\ \text { FAA } & \text { Federal Aviation Administration } \\ \text { FOV } & \text { Field-of-View } \\ \text { Gbps } & \text { Gigabits per second } \\ \text { GPS } & \text { Global Positioning System } \\ \text { IMU } & \text { Inertial Measurement Unit } \\ \text { INS } & \text { Inertial Navigation System } \\ \text { IR } & \text { InfraRed (light spectrum) } \\ \text { IVSS } & \text { Intelligent Visual Surveillance Systems } \\ \text { METAR } & \text { MEteorological Terminal Aviation Routine Weather Report } \\ \text { MP } & \text { Megapixels } \\ \text { MTF } & \text { Modulation Transfer Function }\end{array}$

Send correspondence to C.M:

E-mail: cyrus.minwalla@nrc-cnrc.gc.ca, Telephone: 16139493716 


$\begin{array}{ll}\text { NVG } & \text { Night Vision Device } \\ \text { PSF } & \text { Point Spread Function } \\ \text { ROI } & \text { Region of Interest } \\ \text { SAA } & \text { Sense-and-Avoid } \\ \text { SAR } & \text { Synthetic Aperture Radar } \\ \text { SBC } & \text { Single Board Computer } \\ \text { SNR } & \text { Signal To Noise Ratio } \\ \text { TCAS } & \text { Traffic Alert and Collision Avoidance System } \\ \text { UAS } & \text { Unmanned Aerial System(s) } \\ \text { VFR } & \text { Visual Flight Rules } \\ \text { VMC } & \text { Visual Meteorological Conditions }\end{array}$

\section{INTRODUCTION}

Market forecasts predict that there will be significant growth in unmanned aircraft sales and use over the next decade, and the segment poised for the most explosive growth is in civil and commercial applications using small systems weighing under $25 \mathrm{~kg}$ (Mendelson 2014). Commercial applications such as infrastructure inspection, precision agriculture and audio-visual media production have been very successful since they can be performed locally within visual line of sight of the pilot. However, longer range operations such as power-line inspection or forest fire monitoring are limited owing to the regulatory requirement to see and avoid conflicting air traffic. For unmanned aircraft systems (UAS) to operate beyond visual line of sight of the remote pilot, a "Sense-And-Avoid" (SAA) system with an equivalent level of safety to the manned see-and-avoid system must be employed. The SAA problem has been the subject of a vast amount of academic, commercial and regulatory activity.

\subsection{Roles of an SAA System}

A sense-and-avoid (SAA) system can be decomposed into five general functions: (1) Status communication (2) Determination of traffic that may be in conflict (3) Evaluation of the deconflicting flight path (4) Avoidance maneuver (5) Determination of 'clear of conflict'. The determination of potentially conflicting traffic encompasses the essence of the sense component of the SAA system. The fundamental elements of this function include scanning for potential threats, evaluation of closure rates of detected traffic and the tracking of detected aircraft.

Traffic conflicts must be detected for both cooperative and non-cooperative aircraft. A cooperative system relies upon transmissions from other aircraft to establish traffic position and velocity, whereas a non-cooperative system does not require any special equipment to be installed on the intruder aircraft. Traffic detection can be further classified into passive or active techniques within the cooperative or non-cooperative environment (Table 1).

Table 1. Collision Detection Solution Space

\begin{tabular}{|l|c|c|}
\hline & Cooperative & Non-cooperative \\
\hline Active & TCAS, ADS-B & Radar, LIDAR, SAR, Sonar \\
\hline Passive & - & Electro-Optical (EO), IR, Acoustic, Passive Radar \\
\hline
\end{tabular}

The passive, non-cooperative scenario, which is of interest for this work, is most analogous to human vision (Ebdon and Regan 2004, Griffith and Kuchar 2008, McCalmont et al. 2007). An SAA system in this scenario relies upon a sensor to detect the azimuth and elevation of incoming traffic. The fundamental advantages of this technique are its low cost, and the ability to detect non-transponder equipped traffic. The prime disadvantages include the inability to obtain direct range or closure information, and poor performance in instrument meteorological conditions (IMC).

\section{VISION-BASED APPROACHES TO SAA}

Collision detection via a distributed camera system falls under the broad class of intelligent visual surveillance systems (IVSS) (Kim et al. 2010)(Raty 2010). Where processing activities are localized, the scheme can be 
referred to as an Embedded Distributed Smart Camera (EDSC) network (Shen 2012). Such a system can theoretically encompass hundreds of cameras in a small form-factor, such as the AWARE-2/ARGUS-IS(Brady et al. 2012). Commercially available multi-megapixel image sensors, equipped with plastic aspheric optics, have good responsivity, noise characteristics and angular resolution (McGuire 2010), exceeding the resolution and noise requirements for an EO SAA system (Grilley 2005).

Vision-based approaches applicable to the sense-and-avoid problem, utilizing a combination of image processing and machine learning techniques, are well-documented in literature. Forlenza et al (Forlenza et al. 2012) describe an air-to-ground target detection approach implemented in hardware on an unmanned helicopter. They compared the Harris-Stephens-Plessey and Shi-Tomasi feature detectors, albeit with synthetic targets. Dey et al (Dey et al. 2009; 2011) present novel approaches for aircraft detection tested against multiple sensor and lens configurations, a processing pipeline utilizing morphological filtering and trained classifiers, albeit the camera platform is ground-based and observes a quasi-static background, which is not representative of airborne sense and avoid. Fasano et al (Fasano et al. 2014; 2008) have numerous conducted airborne collision trials with fused sensor methodologies (EO/IR, EO/Radar). Target detection and tracking algorithms were tested in real-time with encouraging results. Lai et al (Lai et al. 2013) conducted airborne tests with EO sensors and an image processing pipeline utilizing morphological filtering techniques.

\subsection{Contributions}

Evaluation of SAA systems is a complex process spanning modeling, simulation, static and dynamic (flight) tests or combinations thereof. Field evaluation, in the form of flight tests, is vital to the proper assessment of any aircraft detection instrument. In this work, the following contributions are proposed: A metric for denoting the range performance of an EO system is proposed. This metric, labeled as the "range at first detection" $\left(R_{0}\right)$, can be extracted directly from in-flight imagery via a technique that is robust against target fluctuations, host and target platform oscillations, scene non-uniformity, atmospheric scattering, rolling shutter artifacts and false alarms. Since the metric is derived directly from empirical observations, the need to model the sensor, the target, and any intermediate transfer functions was obviated, migrating the methodology from a model-dependent paradigm to a measurement-dependent one. A prototype EO camera array, DragonflEYE, was developed and evaluated in actual collision-course flights with a UAS surrogate and manned intruders. DragonflEYE is a network of smart camera nodes assembled from commercially available components. Such an array allows the optics to span an extensive non-spherical field of view while retaining a small, uniform optical point-spread function (PSF). In addition, selective sampling of the field of view in resolution, coverage and overlap, can be performed. Overall, this evaluation serves to determine whether a passive EO sensor can satisfy the minimum time-to-detection requirement of SAA systems. It is postulated that the described techniques may be extended to other similar sensing modalities, such as IR, SAR or NVG systems.

\section{MEASUREMENT METHODOLOGY}

The methodology and measurement of the signals obtained from intruders on a potential collision course are presented in this section. Of interest are the signal, background and noise characteristics of potential targets, as well as the atmosphere, motion (platform/intruder) transfer functions. These definitions are used to derive the principal figures of merit, namely the range at first detection $\left(R_{0}\right)$ and the time to impact $\left(t_{0}\right)$ which can be used to evaluate any collision sensing instrument. It is noted that $R_{0}$ and $t_{0}$ represent the upper bound on the range and time available for the successful identification of potential collision course targets. As such, they serve to define the total time available for any processing activities required to estimate a collision threat, plan an avoidance maneuver and either execute said maneuver or communicate a warning to the pilot. Estimating collision threats from detected targets is relegated to future work.

\subsection{Operating Environment}

The scenario consists of the host platform, housing the sensor, operating in a day-time environment, with one or more potential intruders in the scene. Both the intruder and the host platform are expected to be in level, non-maneuvering and non-evasive flight, operating at general aviation altitudes under VMC and away from local aerodromes. Potential intruders were expected to emerge from the far field, at or near the horizon, also in 
level flight. The collision, therefore, is a byproduct of the spatio-temporal intersection of the host and intruder aircraft following their respective flight-plans. A collision course represents any flight geometry that may bring both aircraft within 500 feet laterally or vertically during the course of the flight, referred to as a "near-miss" collision in aerospace nomenclature. If the two aircraft are on a level collision course, then the azimuth and elevation of the intruder, from the perspective of the host, will remain fixed for the duration of the course. In addition, both intruder and host aircraft are expected to maintain a fixed separation from the cloud ceiling for the duration of their flight paths, to eliminate circumstances where either aircraft may suddenly emerge from or disappear into the cloud ceiling.

\subsection{Signal and noise of the intruder aircraft}

The principal source of scene radiance is diffuse, incoherent illumination from the sun. A typical output power of $\sim 100 \mathrm{~W} / \mathrm{m}^{2}$ can be measured on the Earth's surface on a sunny day. Therefore, it is assumed that incident radiance on the detector plane from the intruder is solely a function of the reflected solar radiance, and not influenced by intruder emissions or third-party artificial sources. Note that although the visible spectrum is assumed, the methodology outlined herein can be extended to other wavelengths and sensing modalities. The cloud ceiling is expected to be in excess of $500 \mathrm{ft}$ vertically from both aircraft. Topographical features are expected to be minimal and solar or scene obscurations are not considered. In addition, the time of day determines the sun elevation angle and observations close to the sun are generally avoided.

\subsubsection{Theoretical SNR}

The signal-to-noise ratio (SNR) is the principal measure of a salient feature in an image. An approximate, theoretical single-pixel signal-to-noise ratio $\left(S N R_{t}\right)$ for a point-like target embedded in the background, in the presence of transfer functions and noise sources, can be defined as per Eq. 1 (Kopeika 1998).

$$
S N R_{t} \approx \frac{\left|M_{t} i_{t}-M_{b} i_{b}\right| \cdot t_{i}}{\sqrt{\left(i_{t}+i_{b}\right) \cdot t_{i}+\sigma_{r}^{2}+\sigma_{r s}^{2}+\sigma_{t g t}^{2}}}
$$

where $M_{t}$ and $M_{b}$ are the modulation transfer functions of the target and background respectively, while $t_{i}$ is the integration period. The radiance from a localized intruder at range $R$ can be approximated as the sum of incoherent intensity contributions from planar differential elements illuminated by sunlight. Noise is encapsulated in the denominator as a combination in quadrature of shot $\left(\sigma_{s}=\sqrt{\left(i_{t}+i_{b}\right) \cdot t_{i}}\right)$, read $\left(\sigma_{r}\right)$ and reset $\left(\sigma_{r s}\right)$ noise elements (Janesick 2001). An additional empirical term, $\sigma_{t g t}$, has been added to account for fluctuations in target radiance.

The image from a real aircraft (Fig. 1a) is generally not a uniform point source, and contains both bright and dark elements compared to the local background scene. Besides the variations in reflectivity due to surface composition and roughness, parts of the aircraft are often obscured from direct illumination, as illustrated in Fig. 1c and Fig. 1d. The signal profile can be sensitive to the orientation of the intruder with respect to the solar direction. At longer ranges, the system point spread function mixes these target components with the local background. Specular reflections can be present at all ranges and contribute to the dynamic variability of the observed signal.

\subsubsection{Empirical SNR}

The above measure for SNR is unwieldy to evaluate from imagery alone. Therefore, we utilize an empirical single-pixel signal-to-noise ratio metric, $S N R_{e}$, which can be computed for any region of interest on any image without external information. Even though the intruder and background have spatial extent, $S N R_{e}$ is structured to look like a single-pixel event against a uniform background. This forces the problem into a standard format by providing a detection criterion independent of the size or complexity of the intruder (Kopeika 1998). Eq. 2 represents $S N R_{e}$ as a direct analog to $S N R_{t}$.

$$
S N R_{e}=\frac{\left|d_{s}-d_{b}\right|}{d_{n}}
$$




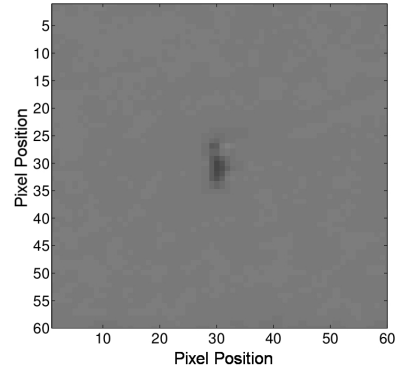

(a) Intruder ROI

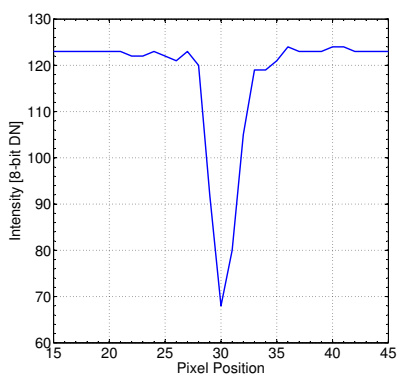

(c) Vertical intensity profile

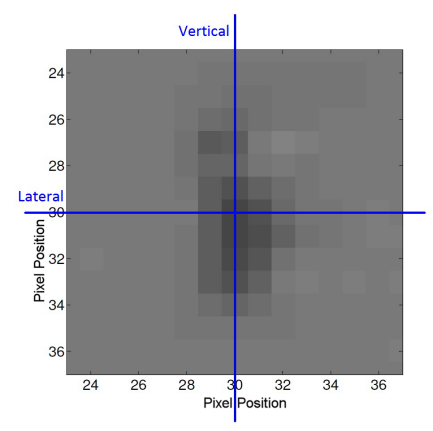

(b) Magnified Intruder

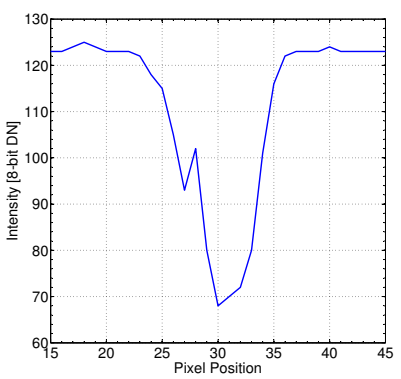

(d) Lateral intensity profile

Figure 1. Crop of a Bell 206 Jet-ranger observed at $3.5 \mathrm{~km}$ with intensity profile cross-sections along the central vertical and lateral axes.

Here, $d_{s}$ is the equivalent target pixel intensity value, while $d_{b}$ is the local background signal estimated at the target pixel. In practice, both $d_{b}$ and $d_{n}$ were, to first order, independent of range between intruder and host. The absolute value operator forces $S N R_{e}$ to be non-negative even though the effective target signal might be weaker than the background. This empirical technique does not require prior knowledge of the intruder, the scene or other imagery, generates reproducible detections and is insensitive to specular reflections or other signal dynamic range constraints. In the limit of an isolated, single-pixel target, $S N R_{e}$ is assumed to be approximately equivalent to $S N R_{t}$ and used in the remainder of the paper. Specular reflectance may sporadically cause spikes in $i_{t}$, which should be observable in the digital number $d_{s}$. Attitude variations of the host and intruder platforms affect signal localization, but not signal strength, assuming that inter-pixel errors such as fixed pattern noise (FPN) and photo-response non-uniformity (PRNU) are minimal and/or partially self-corrected within a commercial sensor.

\subsubsection{Range at first detection - $R_{0}$}

The range at first detection, $R_{0}$, is explicitly defined as the range value at which the empirical SNR crosses a predefined detection threshold, $S_{t h}$. As a figure of merit, $R_{0}$ has the benefit of a simple link to measured quantities, and its random error, spread over an entire collision course flight, can be precisely determined from the uncertainties in the parameters of the fitted $S N R_{e}$ curve.

Individual values of the SNR, corresponding to specific images, are highly susceptible to noise. Let us define a function $S N R_{e}=f(R \mid \overrightarrow{\mathbf{a}})(f(R)$ for convenience) that represents a fit to the empirically acquired SNR values from a single flown collision course, where the independent variable $R$ denotes range. The vector $\overrightarrow{\mathbf{a}}$ contains $n$ fit parameters, enumerated as $\left[a_{i}, a_{2}, \ldots, a_{n}\right]$. Ideally, $n<<N$, where $N$ is the number of samples utilized to perform the fit, allowing $a_{i}$ to be computed simultaneously in an over-determined, least-squares fashion. A good $f(R \mid \overrightarrow{\mathbf{a}})$ fit will lead to reduced parametric $(\overrightarrow{\mathbf{a}})$ uncertainty. Therefore, the optimization function may be structured to find the simplest model with the best fit. Since the SNR is expected to be influenced most strongly 
by the intermediate transfer functions (e.g. atmosphere, optics, motion), parametric models of those can serve as good starting points.

Let us assume that $f(R \mid \overrightarrow{\mathbf{a}})$ derived in this fashion is a smoothly continuous, invertible function over the range of interest. Then, the range at first detection, $R_{0}$, and its associated error, $\delta R_{0}$, can be quantified as per Eqs. 3 and 4 respectively.

$$
\begin{aligned}
R_{0} & =\left.f^{-1}\left(S_{t h} \mid \tilde{\mathbf{a}}\right)\right|_{S N R=S_{t h}} \\
\delta R_{0} & =\sqrt{\sum_{i}^{n}\left(\frac{\partial f(R)}{\partial a_{i}} \delta a_{i}\right)^{2}}+\delta R_{s y s}
\end{aligned}
$$

Note that Eq. 4 is derived from the calculus of errors, giving the random error in $R_{0}$ from the contributions of each fit parameter $a_{i}$. The presence of systematic errors is denoted as $\delta R_{s y s}$. These systematic errors account for network latencies, time-stamp capture and storage offsets, as well as quantization errors inherent in any digital system. Note that the form of $f(R \mid \overrightarrow{\mathbf{a}})$ is germane only with respect to the uncertainties $\left(\delta a_{i}\right)$. It is observed that the empirical nature of $S N R_{e}$ makes it susceptible to systematic errors. We can compare the resulting $R_{0}$ with visual observations to validate the definition of $S N R_{e}$.

\subsubsection{Time to collision - $t_{0}$}

A direct corollary of the range at first detection is the estimated time-to-collision metric, $t_{0}$. The U.S. FAA defines a "collision distance", $R_{m}$, of $500 \mathrm{ft}(150 \mathrm{~m})$. We then define $t_{0}$ to be the time until the intruder is within $500 \mathrm{ft}(150 \mathrm{~m})$ of the host platform. The FAA estimates that a typical pilot has the reaction time of 12.5 seconds to avoid a collision (Federal Aviation Administration (FAA) 1983). We define a parameter called the "minimum time-to-evade", $t_{m}=12.5 \mathrm{~s}$, treating this as the lower bound on the time to collision, such that $t_{0}>t_{m}$ to successfully avoid a collision. Eqs. 5 and 6 compute $t_{0}$ and the associated error respectively.

$$
\begin{gathered}
t_{0}=\frac{R_{0}-R_{m}}{\overline{v_{c}}} \\
\delta t_{0}=t_{0} \cdot \sqrt{\left(\frac{\delta\left(R_{0}-R_{m}\right)}{R_{0}-R_{m}}\right)^{2}+\left(\frac{\delta \overline{v_{c}}}{\overline{v_{c}}}\right)^{2}} \approx t_{0} \cdot \frac{\delta R_{0}}{R_{0}}
\end{gathered}
$$

Here, $R_{0}$ is in units of kilometres, while $\overline{v_{c}}$ is the average closing rate between the two aircraft. Given the GPS-correlated range values per acquired image, $\overline{v_{c}}$ was accurately estimated from a linear fit to a range against time curve. Since $t_{0}$ is a function of the closing rate, the emphasis is placed on the estimation of $R_{0}$. With a known $R_{0}$, a worst-case $t_{0}$ can be estimated by assuming the maximum closing rate of $v_{\max }=250 \mathrm{~m} / \mathrm{s}(500$ knots), obtained by having the host and intruder on a head-on collision course and flying at the maximum GA speed of 250 knots.

\subsection{Intermediate Transfer Functions}

Transfer functions limit the maximum resolution and contrast at the output in imaging systems. In aerial imaging systems, the combined modulation transfer function can be treated as a linear convolution of atmospheric $\left(M_{A}\right)$, platform motion $\left(M_{V}\right)$ and optical $\left(M_{O}\right)$ contributions. Note that $M_{O}$ is static (but can be angle-dependent), $M_{V}$ is a function of time and $M_{A}$, a function of range(Kopeika 1998).

$$
M=M_{A}(R) * M_{V}(t) * M_{O}
$$

For collision course intruders, range and time are linked via the closing speed, therefore $M_{A}$ is expected to behave monotonically with respect to time. Atmospheric effects may dominate for range performance evaluation. Failure to observe monotonic behaviour in $M_{A}(R)$ can prove valuable in determining the validity and probability of a collision. It is observed that $M_{A}(R)$ will be different between the target and the background. 


\subsubsection{Atmosphere - $M_{A}(R)$}

Atmospheric scattering adversely affects the observed intruder signal. If the atmosphere is modeled as a finite, homogeneous medium with uniform particulate radius and density, the impact of absorption and scattering may be approximated by the Beer-Lambert Law(Overington 1976) (Eq. 8),

$$
I_{\text {out }}=I_{0} e^{-\sigma_{e} R}+B_{0}\left(1-e^{-\sigma_{e} R}\right), \text { where } \sigma_{e}=b_{r}+\tau
$$

where the output $\left(I_{o} u t\right)$ is a function of the input intensity $\left(I_{0}\right)$ from a small target exponentially attenuated and merged with the local background signal $\left(B_{0}\right)$ due to absorption and scattering. Here, $\sigma_{e}$ is the atmospheric extinction coefficient, decomposed into scattering $\left(b_{r}\right)$ and absorption $(\tau)$ coefficients. Scattering is expected to dominate, such that $\tau \approx 0$. Note that the local background signal $\left(B_{0}\right)$ is typically a function of range, however, for long-distance imaging, $R>>f$, background irradiance is approximately constant for a given attenuation coefficient. The extinction coefficient can be linked to atmospheric visibility by Koschmeider's formula (Eq. 9) for horizontally viewed targets, where $V$ is the visibility in kilometres (Overington 1976).

$$
\sigma_{e} \simeq \frac{3.912}{V} \quad(\text { for } \lambda=550 \mathrm{~nm})
$$

An initial estimate for local visibility can be obtained from the local aerodrome METAR profile. Since prevailing local conditions may vary significantly, the METAR forecast served mainly as a "go/no-go" condition for flight tests. Given the wide field-of-view specification, the sun may often be in the direct field of view of one or more camera nodes. Immediate effects include saturation, blooming, retro-reflections and lens flare, while long-term exposure may generate excess heat, resulting in increased dark current or undesired photo-response non-uniformity. Long-term ultraviolet radiation can cause permanent changes in the substrate doping, leading to burn-in (large drain current), increased cross-talk and undesired photo-response non-uniformity.

\subsubsection{Platform motion - $M_{V}(t)$}

Aircraft are prone to dynamic changes in attitude, position and their higher order derivatives from on-board propulsion mechanisms and wind effects (gusting). Flight dynamic modes dominate (Etkin and Reid 1995), contributing low-frequency, high-amplitude oscillations that are coupled across the control surfaces. Mechanical vibrations are high-frequency, low-amplitude artifacts that are generated at the mount's resonant frequency. Hence, aircraft-mounted cameras are subjected to linear, sinusoidal and random motion simultaneously (Holst 2003). The resulting image artifacts are frequently termed 'shear', 'skew' or 'wobble', however, the net result is always target blurring. Consider Eq. 10, a combination of three MTFs: The first term models linear motion as a $\operatorname{sinc}()$ function, where $\omega_{l}$ is the spatial sampling frequency, $v_{l}$, the linear velocity, and $t_{i}$, the integration time. The second term details oscillatory motion, where the equivalent blur radius is twice the amplitude $\left(a_{s}\right)$ of the oscillatory frequency $\left(\omega_{s}\right)$. Finally, random motion is best approximated by a Gaussian distribution of standard deviation $\left(\sigma_{r}\right)$ as the RMS random displacement.

$$
M_{V}=M_{l i n} * M_{s i n} * M_{r n d}=\left|\operatorname{sinc}\left(\frac{\omega_{l}}{2 \pi} v_{l} t_{i}\right)\right| * J_{0}\left(a_{s} \omega_{s} D\right) * e^{-\sigma_{r} \omega_{r}^{2}}
$$

For outdoor scenes illuminated by sunlight, adequate irradiance is incident on the detector to set a sufficiently short exposure time such that intra-scanned regions (singular rows) are unaffected by the host platform motion. In brief, motion blur is obviated with a fast shutter speed. This assumption was experimentally verified for the present case where an adequately exposed image without motion blur could be obtained by a shutter speed of $1 / 2000$ seconds and a lens aperture of $\mathrm{f} / 4.0$, and should hold true for high visibility aerial scenes. However, it is noted that the acquisition process is still prone to inter-scan artifacts due to platform motion, such as rolling shutter wobble, shear or line-scan artifacts. In addition, small attitude oscillations in the target combined with the sun angle can affect the perceived location and intensity of the received signal. No explicit correction was made during acquisition, therefore it is expected to be present as an additional source of error in the acquired data. 


\subsubsection{Optics - $M_{O}$}

The optical transfer function can be decomposed into diffraction, aberration, distortion and defocussing components (Eq. 11).

$$
M_{O}=M_{\text {diff }} * M_{\text {aber }} * M_{\text {distort }} * M_{\text {defocus }}
$$

Lens diffraction $\left(M_{\text {diff }}\right)$ is the fundamental lower limit to optical spatial transfer functions, and is commonly defined by the Fraunhofer diffraction pattern, or Airy pattern. The intensity $I$ is defined as a function of the radial distance (Eq. 12) for a fixed wavelength, focal ratio and incident irradiance (Born and Wolf 1999).

$$
I(r)=I_{0}\left(\frac{2 J_{1}(x)}{x}\right), \quad \text { where } x=\frac{\pi r}{\lambda F}
$$

where $J_{1}$ is the first-order Bessel function, $I_{0}$ is the maximum intensity (usually normalized), $\lambda$ is the wavelength, $F$ is the focal ratio of the lens, and $r$ specifies the radial distance from the optic axis. In most applications, the width of the main lobe is of particular interest, and the half-maximum occurs at $x \approx 1.62$. The disc may be approximated by a Gaussian function, where the full-width-at-half-maximum (FWHM) is related to the standard deviation $\sigma$ by $F W H M=2 \sqrt{2 \ln (2)} \sigma$. Alternatively, for a given lens of known focal ratio, the half-width of the Airy disc may be approximated since $\sigma \approx 0.45 \lambda F$.

Although diffraction is the lower limit, typical lenses exhibit spheroid aberrations $\left(M_{\text {aber }}\right)$, distortion $\left(M_{\text {distort }}\right)$ and astigmatic artifacts, especially in inexpensive commercial C-mount lenses. These aberrations are welldescribed by multi-degree polynomial formulations, such as the Zernike equations (Mahajan 1994). Spherical and chromatic aberrations, if significant, may require an explicit deconvolution of the point-spread function (PSF). Distortion does not impact the signal, rather it affects the measured azimuth and elevation of the target. It is commonly corrected ex tempore by Brown's infinite Taylor series distortion model(Brown 1971) as per Eq. 14.

$$
\begin{gathered}
x_{u}=x^{\prime} \cdot\left(1+k_{1} r^{2}+k_{2} r^{4} \ldots\right)+t_{1}\left(r^{2}+2\left(x^{\prime}\right)^{2}\right)+2 t_{2}\left(x^{\prime} y^{\prime}\right)\left(1+t_{3} r^{2}+\ldots\right) \\
y_{u}=y^{\prime} \cdot\left(1+k_{1} r^{2}+k_{2} r^{4} \ldots\right)+t_{2}\left(r^{2}+2\left(y^{\prime}\right)^{2}\right)+2 t_{1}\left(x^{\prime} y^{\prime}\right)\left(1+t_{3} r^{2}+\ldots\right) \\
\text { where } x^{\prime}=x_{d}-x_{c}, \quad y^{\prime}=y_{d}-y_{c}, \text { and } \quad r=\sqrt{x^{\prime 2}+y^{\prime 2}}
\end{gathered}
$$

Here, $\left(x_{u}, y_{u}\right)$ represent the undistorted version of the distorted point $\left(x_{d}, y_{d}\right)$, while $\left(x_{c}, y_{c}\right)$ is the principal point on the optical plane. It is sufficient to consider the first two radial $\left(k_{1}, k_{2}\right)$ and tangential $\left(t_{1}, t_{2}\right)$ coefficients for most lenses. Since all optical artifacts are static, it is sufficient to characterize each lens-imager pair in advance of flight tests.

\section{FLIGHT TEST METHODOLOGY}

Flight tests were conducted by two co-altitude, non-maneuvering aircraft flying collision course trajectories in clear sky conditions with maximum visibility. A day of flights consisted of 10-20 "trials", where each trial represents a single fixed collision geometry flown from maximum separation to the "near-miss" distance. Each trial was terminated by aircraft maneuvers in preparation for the next trial.

\subsection{Instrument}

The DragonflEYE concept was developed at the Vision Sensor (VISOR) Laboratory as a low-distortion, widefield-of-view prototype aircraft detection sensor (Fig. 5.1.1) consisting of identical smart camera nodes (Fig. 2c). The nodes are designed for in-situ processing of raw imagery to circumvent bandwidth limitations. A similar EO instrument, Cerberus (not pictured here), was developed at the National Research Council Canada consisting of a 3-camera array sans per-camera processing elements (Table 4). 


\begin{tabular}{|l|l|c|c|}
\hline Component & Parameter & Value & Units \\
\hline \multirow{4}{*}{ Flight } & Topology & $5 \times 2$ & nodes \\
Prototype & FOV & $30 \times 9$ & $\mathrm{deg}$ \\
& Mass & 50 & $\mathrm{~kg}$ \\
& Power & 75 & $\mathrm{~W}$ \\
& Network & 1.0 & $\mathrm{Gbps}$ \\
\hline \multirow{5}{*}{ Smart } & Pixel count & $1600 \times 1200$ & $\mathrm{pix}$ \\
& ARM A8 & 600 & $\mathrm{MHz}$ \\
& Network & 100 & $\mathrm{Mbps}$ \\
& Mass & 0.5 & $\mathrm{~kg}$ \\
& Framerate & $2.5 \pm 0.3$ & $\mathrm{fps}$ \\
\cline { 2 - 4 } & f & 25 & $\mathrm{~mm}$ \\
& dpix & 2.6 & $\mu \mathrm{m}$ \\
& Spatial res. & 0.1 & $\mathrm{mrad}$ \\
& Dyn. Range & 8 & $\mathrm{bit}$ \\
& $1 / f$ & $5.6 \pm 0.5$ & $\mathrm{stop}$ \\
& Exposure & $0.2 \pm 0.02$ & $\mathrm{~ms}$ \\
\hline
\end{tabular}

(a) Hardware parameters of the flight prototype

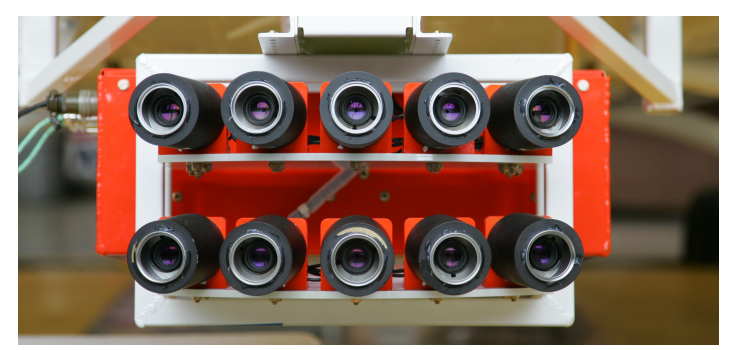

(b) Front view of nodes

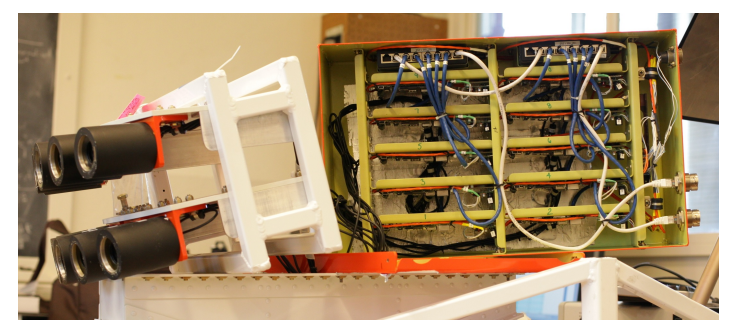

(c) Side view of SBCs in a weather-sealed housing

Figure 2. DragonflEYE flight prototype specifications and illustrations. Camera nodes and networked SBCs in a weathersealed housing fastened to the mounting plate. Each node could be individual adjusted in the horizontal, while a single row was adjusted simultaneously in the vertical.

\subsubsection{System Topology}

Each node is configured with a 2 megapixel Logitech C600 webcam, a $25 \mathrm{~mm}$ C-mount lens, and an embedded single-board ARM computer (SBC) (Minwalla et al. 2012). The camera nodes were linked by an Ethernet backplane. For the flight prototype, the number of camera nodes was restricted to allow the read-out of raw images to an external storage device at a useful frame rate $(2.5 \mathrm{~Hz})$. As such, the prototype had ten cameras in a $5 \times 2$ configuration (with overlap) for a total viewing frustum of $30 \times 9$ degrees at the full angular resolution of $0.1 \mathrm{mrad}$ per pixel. Additional parameters of the array are available in Table 2a. An on-board rack-mount computer, equipped with two network interface cards, was configured as both a "test node" and a "world node". As a test node, it served as the endpoint for raw image capture and storage. As a world node, it served as the gateway between the aircraft and the instrument. The instrument connected to the test node via a 1.0 Gbps Ethernet link, while the smart nodes internally linked at 100 Mbps. A separate $100 \mathrm{Mbps}$ link connected the rack-mount PC to the aircraft. Electrical power was provided by the host aircraft. Separate power conversion modules, SBCs, ancillary hardware and software were allocated to each camera node, so that a failure in one node would not compromise the operation of others.

The prototype footprint was intentionally large for flexibility of configuration and replacement of COTS components. It is expected that embedded optics and custom electronics are straightforward to achieve, and will result in dramatic size and mass savings. Details regarding hardware/software architecture, calibration, installation and airworthiness are covered in archival work(Minwalla 2013) and are eschewed here for brevity. The technique described herein is generic and should be applicable to all EO systems regardless of image sensor and lens configuration, hardware/software architecture or specific calibration parameters. The only specific requirement is that the system is synchronized to the ownship INS, and the precision of synchronization will contribute to the measured $R_{0}$ value.

\subsubsection{Data Acquisition}

Although the nodes were designed to perform in-situ processing, they were reconfigured in a hierarchical fashion for these flight tests to expedite the recording of raw frames. As a result, bandwidth constraints limited the total system acquisition time to $2.5 \mathrm{~Hz}$ for ten nodes. All nodes (camera and world) were synchronized to the host aircraft's inertial navigation system (INS). Each acquired image was tagged by a GPS time-stamp with a time uncertainty of $\pm 10 \mathrm{~ms}$. Ancillary data, including host and intruder orientations, altitudes and airspeeds, 


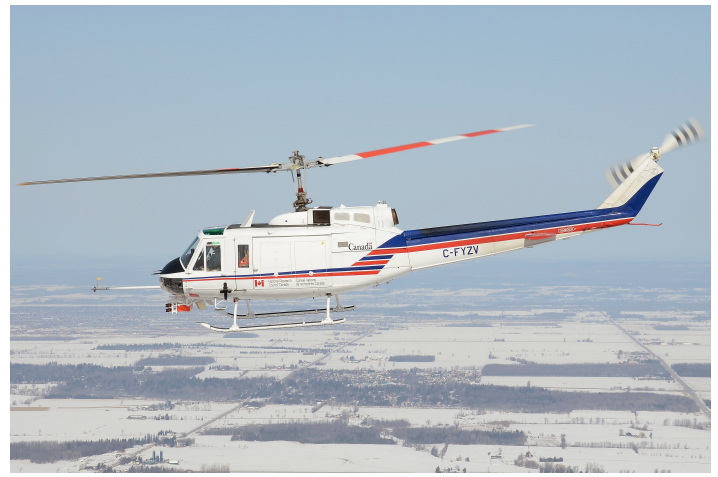

(a) Surrogate UAS - Bell 205

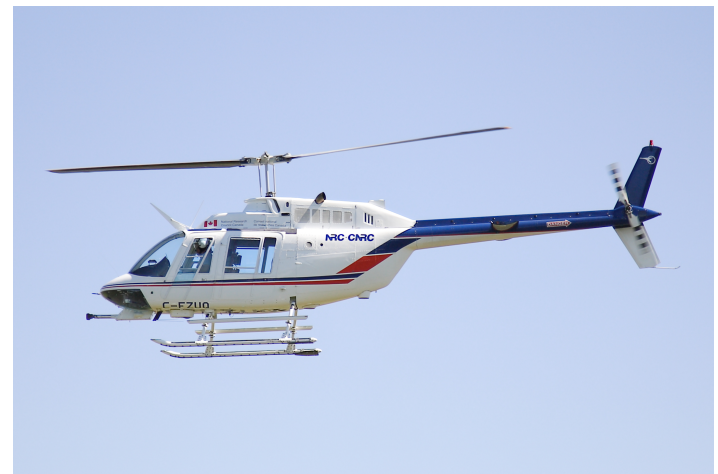

(b) Intruder - Bell 206

Figure 3. Host and intruder aircraft used for airborne flight tests. DragonflEYE (orange) was mounted under the nose in a forward-looking configuration, while Cerberus (black) was mounted on the port side.

were recorded with each frame acquisition. Colour information was available but discarded. Demosaicing was performed in-camera with a manufacturer-supplied algorithm.

\subsection{Aircraft}

Flight tests were conducted in controlled airspace near the Flight Research Laboratory facility at Ottawa International Airport (CYOW). Trials described in the paper were flown on Feb 28, 2012, between 4:00 and 5:00 pm EST and Mar 7, 2012 between 9:00 and 10:00 am. Two helicopters were flown, a Bell 205 (the host platform) and a Bell 206 (the intruder) (Fig. 3). The DragonflEYE instrument was rigidly mounted to the Bell 205, which acted as a surrogate UAS (Ellis and Gubbels 2005). The system topology after DragonflEYE integration is depicted in Fig. 5a.

Each aircraft was instrumented with an automatic dependent surveillance-broadcast (ADS-B) transceiver and an INS (Leach et al. 2003) comprising an inertial measurement unit (IMU), a global positioning system (GPS) unit, and an SBC. A flight test engineer interacted with the instrument via an in-cockpit LCD display and trackball. Maintaining the desired collision trajectory beyond the range of visual acquisition was an important practical consideration, a task assisted by an 'Intercept Display' (Keillor et al. 2011) that provided ADS-B-based bearing and range guidance for the flight crew. The display was implemented on an Apple iPhone, which was fastened to the glare-shield in the intruder (206) and the centre console in the host (205). The DragonflEYE instrument was calibrated in orientation with respect to the host IMU, such that the pointing direction with respect to the aircraft's heading was explicitly known to \pm 0.5 degree. The instrument was activated and initialized on the ground at the start of the flight tests. Given the short window of optimal atmospheric conditions alongside other operational constraints, failures at this stage typically meant a cancellation of the flights for that day.

Conducting near-collision misses with a UAS and manned platform in uncontrolled (Class G) airspace is a high-risk activity due to the complex flight safety requirements and the current state of regulations. Use of the Bell 205 as a surrogate mitigates the risk of a collision while maintaining comparable platform behaviour. In particular, the airspeeds flown by the 205 (40-80 knots) are equivalent to those of a small ( $<25 \mathrm{~kg}$ ) UAS. The 206, with a frontal cross-section area of $\approx 1.0 \mathrm{~m}^{2}$, is representative of a typical intruder (Cessna 172) with a similar cross-section profile encountered in Class G airspace. The size of the host aircraft that carries the prototype sensor does not impact the proposed technique or the test methodology. Challenges regarding integration onto a small UAS are primarily related to size, weight and power (SWaP) requirements. Electro-optical systems, in particular, seem to demonstrate a straightforward path to miniaturization without requiring significant elaboration. A large host aircraft not only permits the carriage of larger, heavier prototype hardware, reducing development time and allowing rapid iteration of development and test, but also enables the simultaneous test and evaluation of multiple prototype instruments. 


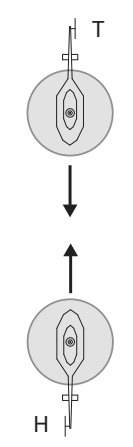

(a) Head

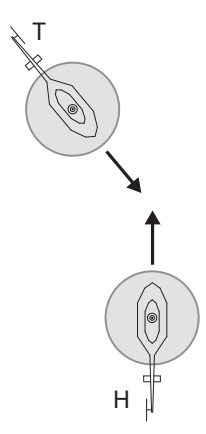

(b) Azimuthal

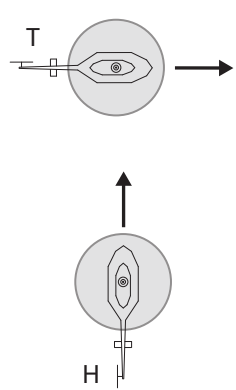

(c) Perpendicular

Figure 4. Schematic representation of collision geometries. Letters ' $\mathrm{H}$ ' and ' $\mathrm{T}$ ' indicate host/surrogate and intruder/target platforms.

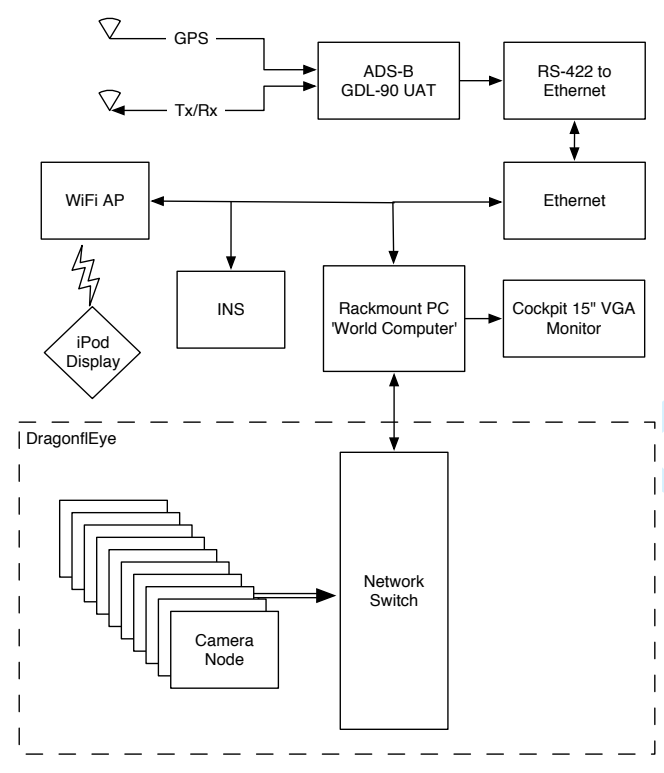

(a) Host platform topology after instrument integration

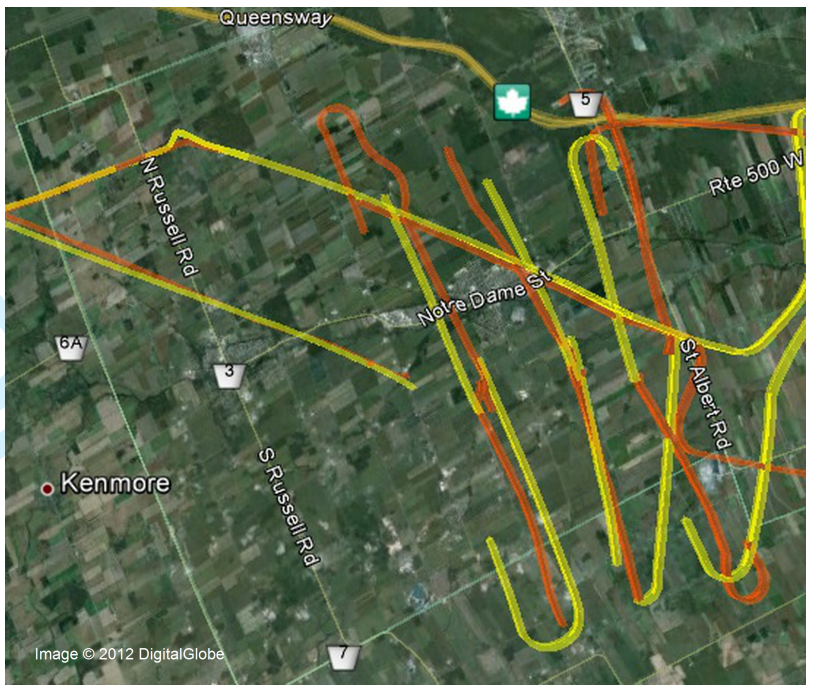

(b) Paths flown by the two aircraft during the Feb 282012 flight trials. The host (Bell 205) flight path is indicated in orange, while the intruder (Bell 206) is indicated in yellow.

Figure 5. Integration of the prototype EO array aboard the UAS surrogate and flown flight paths overlaid onto a terrain map.

\subsection{Flight Trials}

Once the ground tests proved successful, the crew proceeded with flight tests. A total of 15 collision intercept courses were flown on Feb 28, 2012 between 4:00 pm and 5:00 pm EST. All trials started beyond visual range (nominally 10 nautical miles) and relied on the Intercept Display for alignment. Each trial was terminated by aircraft maneuvers in preparation for the next trial. When the flight crew of each aircraft noticed the other, they recorded the distance identified on the intercept display. Both the host and intruder aircraft were flown at a constant airspeed in non-maneuvering flight along a predetermined flight path maintained within the error limits of normal pilot response.

Fig. 4 schematically shows a sub-set of the trials flown. Head-on and azimuthal-offset collisions define the critical operating path for the instrument, and so are emphasized in the analysis. The head-on and azimuthal collision trials were conducted with the host in level flight and the intruder approaching along a well-defined, level collision trajectory. In contrast, perpendicular trials depict a roughly constant-ranged target. Although this is not a collision case, it proved useful for visual cue analysis. Fig. 5b depicts the actual trajectories flown on Feb 28, 2012 near CYOW. Note that additional descending, overtake and descending-overtake trials flown 


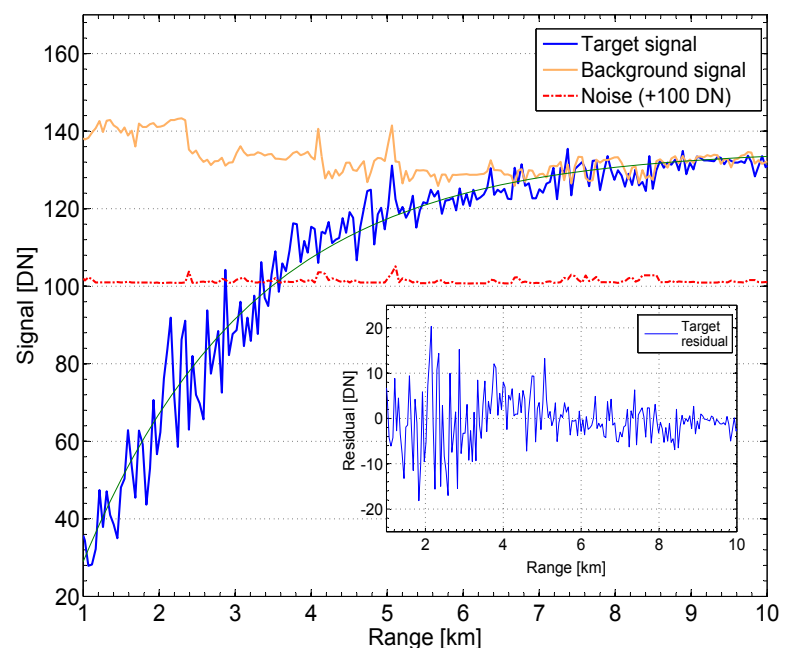

(a) Individual signal, background and noise elements from a head-on flight trajectory. The inset shows the residual for the target signal from the trend whereas noise has been offset by a factor of $100 \mathrm{dn}$ for comparison.

Figure 6. Signal, background and noise measurements of a singular head-on collision trial

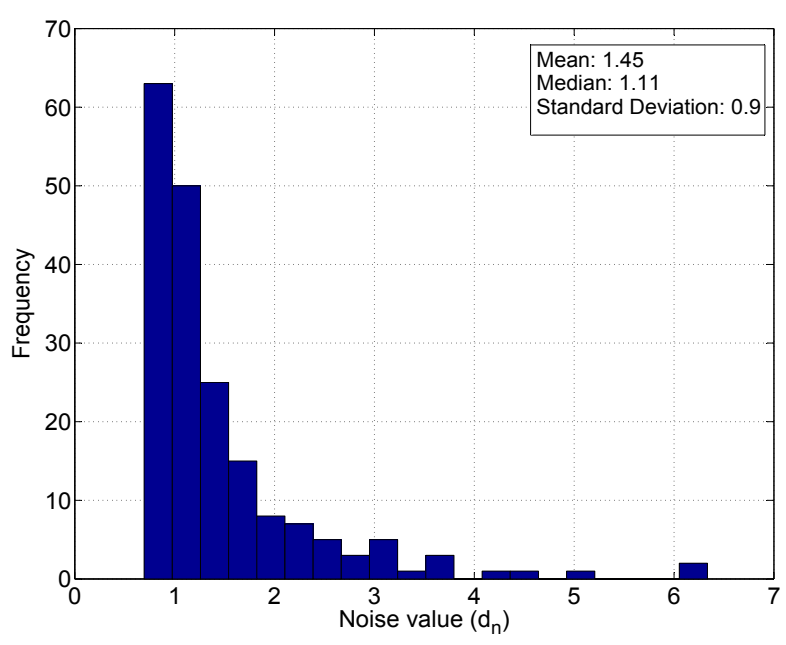

(b) Histogram of noise estimates from one flight trial

that day are not discussed in the paper. During each evaluation trial, image archiving was triggered upon radio confirmation between the two aircraft. Archiving was stopped after the intruder aircraft flew past the host.

\section{ANALYSIS AND RESULTS}

Recall that the metrics of interest are $R_{0}$, the range at first detection, and $t_{0}$, the time to impact. Both quantities rely on $S N R_{e}$ as a function of range, where range is the separation between the host and intruder aircraft, as determined by their on-board GPS instruments. The extraction methodology below explains how a complete set of $S N R_{e}$ values against range values is obtained for each trial.

\subsection{SNR Extraction Procedure}

For each image in which a target was expected, the following post-processing procedure is performed to define its signal, $d_{s}$ : Target regions of interest (ROIs) are manually selected per frame by a person. To facilitate precise detection, the image sequence depicting the trial is incremented in reverse order, starting with an easily visible target in close proximity to the host. The manual selection process trades off optimal detection via automated algorithms for a consistent ground-truth estimate. Then, the pixel location in the ROI with the largest variation from the ROI mean is extracted and treated as the target location. The target signal, $d_{s}$, is then computed by taking the mean of a $3 \times 3$ window centered at this "most-visible" target feature. The averaging operation is intended to treat the complex, disjoint radiance profile apparent in the target as an equivalent single-pixel phenomenon.

The local background signal, $d_{b}$, is calculated as follows: An annular ROI is selected around the target region, and a mean pixel intensity is computed. When this annular ROI contained the horizon, only the segment on the same side of the horizon as the target is used. In the head-on case, the target is usually above the horizon and is seen against a sky background.

An estimate of the random noise, $d_{n}$, is computed by first differencing temporally adjacent local background annuli and then computing the standard deviation of each difference image. The differencing step is utilized to reduce the impact of scene structure. Nonetheless, residual spatial information may still be present. Fig. 6b is a histogram of noise over a sequence of difference images acquired from one trial. The empirical $d_{n}$ includes contributions from read noise, shot noise, reset noise, fixed pattern noise and photo-response non-uniformity (Janesick 2001). Each of the individual contributions was estimated to be $<1$ in units of digital number. Note that $\sigma_{t g t}$ has been explicitly excluded from $d_{n}$ by utilizing the background ROI. 


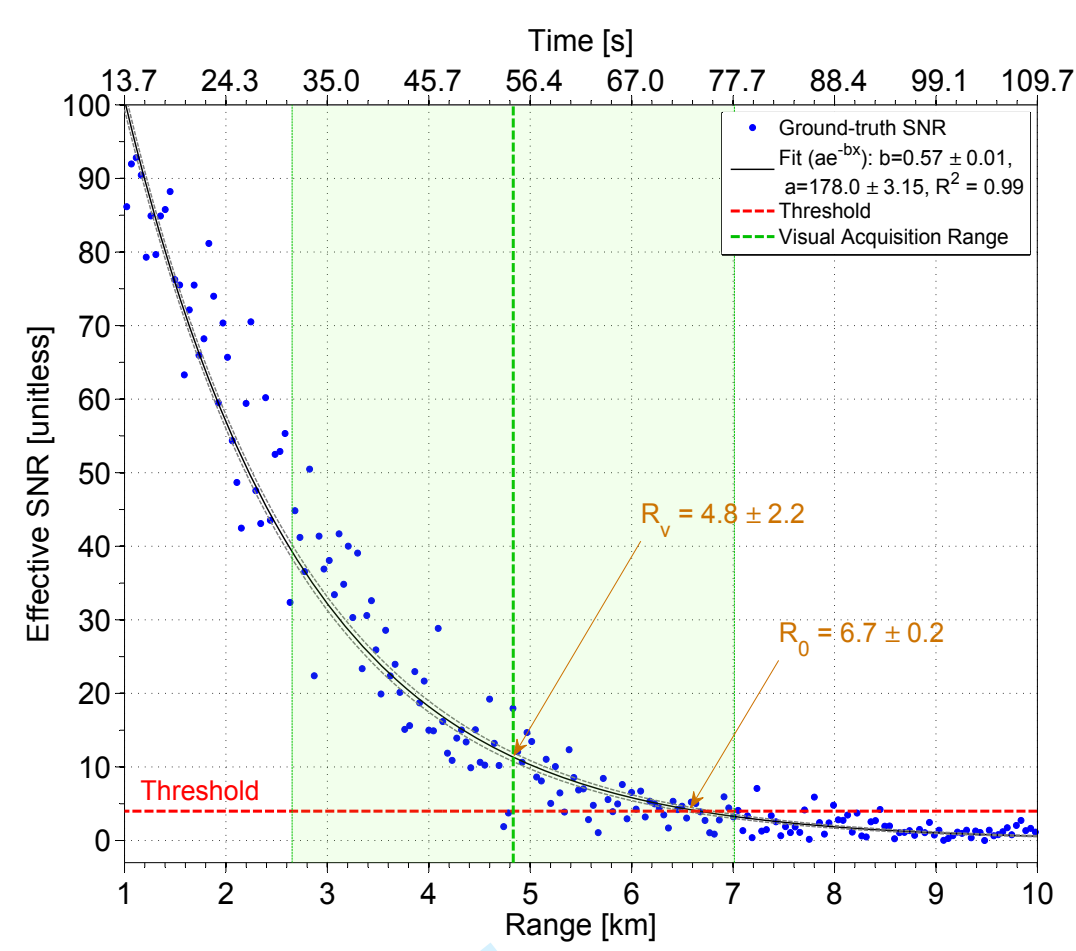

Figure 7. SNR as a function of range and time for a typical co-altitude head-on trial. The raw data is fitted to a twoparameter exponential fit, with parameter values listed alongside 2 -standard deviation bounds. The threshold $(S N R=4)$ and pilot detection range $\left(R_{v}=4.8 \pm 2.2 \mathrm{~km}\right)$ are plotted as dotted horizontal and vertical lines respectively, with two standard deviation bounds indicated for the pilot range.

\subsection{Empirical SNR Measurement}

Fig. 7 shows the $S N R_{e}$ extracted as per Section 6.1 against range for a representative head-on trial. A 'relative' time axis is also shown on the top x-axis. The time origin is set to the range at which the target would cross a "near-collision" boundary (Federal Aviation Administration (FAA) 1983). At extreme ranges, the $S N R_{e}$ values tend to zero, as expected when the target signal vanishes into the background. Each camera node functioned in full auto-exposure mode, placing the most common signal intensity, represented by the average background, at the midpoint of the dynamic range curve. This process halved the dynamic range available to the target. For realistic targets with a spatially disjoint radiance profile, this exposure procedure, although sub-optimal, is deemed acceptable.

The data is fitted empirically to an exponential function of range $\left(f_{e}(R)\right)$, where $b$ is the rate of exponential decay, while $a$ is a scaling factor (Eq. 15).

$$
f_{e}(R)=a e^{-b R}
$$

An error estimate (two standard deviations) is shown for each parameter, with prediction bounds corresponding to these estimates plotted as dotted lines fencing the fit. The fit is precise, with a coefficient of determination value greater than 0.99. Parameters $a$ and $b$ have uncertainties of less than $2 \%$ over two standard deviations, lending credence to the choice of model. It is noted that the choice of the exponential fit is non-unique and the formulation of $R_{0}$ is insensitive to the choice of fit function. However, the exponential model is the model of choice as it explains the data with a minimum number of fit parameters and is the standard model in cases where atmospheric extinction dominates. Note that if we assume the atmosphere to dominate, as per the choice of function, then $b$ is equivalent to $\sigma_{e}$ from Eq. 9, and the theoretical Koschmeider visibility $V=\frac{3.912}{b}=6.86 \mathrm{~km}$. 


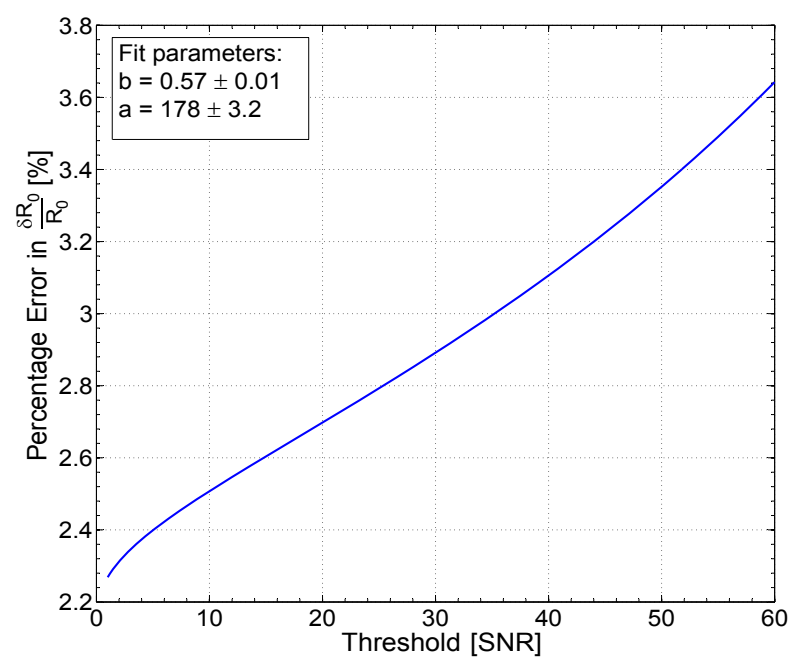

(a) Fractional error, $\delta R_{0} / R_{0}$, as a function of threshold, calculated for the head-on trial depicted in Fig. 7. Note that the fractional error increases with threshold, although the net change is small.

Figure 8. Sensitivity analysis of the range performance evaluation.

\subsection{Measurement of $R_{0}$ and $t_{0}$}

Given the fit function $f_{e}(R)$ and the previous definition of $R_{0}$ (from Section 6.1) as the range value at which the empirical SNR crosses a predefined detection threshold, $R_{0}$ may be computed for the experimental data. In Fig. 7, a horizontal dashed-line indicates $S_{t h}$ chosen at $S N R=4$, which corresponds to a probability of detection $\left(P_{d}\right)$ value of $90 \%$ and a false-alarm rate of $1 \%$ under a Gaussian ergodic process assumption (Holst 2003). $R_{0}$ may then be calculated for $f_{e}(R)$ as per Eq. 16, with the precision derived as per Eq. 17 .

$$
\begin{gathered}
R_{0}=\frac{\ln a}{b}-\frac{\ln S_{t h}}{b} \\
\delta R_{0}=\sqrt{\left(\frac{\delta a}{a b}\right)^{2}+\left(\frac{\delta b}{b^{2}}\left(\ln S_{t h}-\ln a\right)\right)^{2}}+\delta R_{\text {sys }}
\end{gathered}
$$

Here, $a$ and $b$ are the fit parameters from Eq. 15, while $\delta R_{s}$ encapsulates the systematic error in range values. Position values are derived from the INS units installed on both aircraft, with a positioning error of $\delta R_{s y s}=$ $\pm 10 \mathrm{~m}$ or less. For this particular head-on trial, a value of $R_{0}=6.7 \pm 0.2 \mathrm{~km}$ is observed. Note that $\delta R_{s y s}$ is small compared to the total error in $R_{0}$. This value compares favourably to $V=6.86 \mathrm{~km}$ as per the atmospheric extinction model.

Fig. 8a shows the fractional error $\delta R_{0} / R_{0}$ as a function of threshold $\left(S_{t h}\right)$. Although the percentage error is observed to increase with increasing threshold values, the total deviation is less than $2 \%$. Since determination of $R_{0}$ uses the imagery from the entire trajectory of a test flight it results in an averaging of errors. In particular, the large fluctuations in $S N R_{e}$ values (Fig. 7), which strongly affect any visual selection of $R_{0}$, are suppressed. It is hypothesized that this benefit is relatively independent of the details of the experiment or the sensor.

\subsubsection{Visual acquisition range}

The mean visual acquisition range, $R_{v}$, is the range where a typical pilot is expected to spot a target under ideal circumstances. Optimal conditions were guaranteed by the time of day, visibility and the intercept display, the latter focusing the flight crew's look direction to a localized region of the scene, maximizing their chances of spotting the intruder aircraft. These observations were aggregated into the mean visual estimate $R_{v}$ and the associated error. A vertical dashed line in Fig. 7 denotes a value of $R_{v}=4.8 \pm 2 \mathrm{~km}$ with dashed vertical error bars at two standard deviations. Despite the aid of the Intercept Display, a significant variation in the visual 
range was observed. The large variability in $R_{v}$ is expected to be both a function of target signal fluctuations and the psycho-visual characteristics of human agents uncovering small targets in an aerial scene (Andrews 1991). The large error reduces the effectiveness of $R_{v}$ as a comparison metric.

\subsubsection{Time to collision}

The time to collision, $t_{0}$, may be similarly computed as per Section 6.1. For the flight trials in question, the average closing rate is very consistent per trial. In particular, the representative trial in Fig. 7 possessed an average closing rate of $94 \mathrm{~m} / \mathrm{s}$. Given a minimum near-miss distance of $R_{m}=500 \mathrm{ft}(150 \mathrm{~m})$, the time-to-impact and its corresponding error, as per Eqs. 5 and 6, compute to $t_{0}=66 \pm 2 \mathrm{~s}$.

\subsection{Impact of Resolution on $R_{0}$}

The instrument has a measured angular resolution of $0.1 \mathrm{mrad}$. This specification meets the design requirement of filling a pixel with a $1.0 \mathrm{~m}^{2}$ target at $10 \mathrm{~km}$. The flown intruder (Bell 206) cross-section of $1.9 \times 3.5 \mathrm{~m}$ has an angular extent that is five times larger, thereby exposing the potential for resolution-based analysis. Fig. $8 \mathrm{~b}$ depicts the observed target SNR on a sythentically generated sub-resolution image set. Each image in the sequence is subjected to bilinear sub-sampling, and sub-sampling factors of 2 and 4 are tested. Since the sensor size was 2.0 megapixels (MP), this results in images of 1.0 MP and 0.5 MP respectively. Curves are generated by repeating the entire analysis procedure for the sub-sampled versions. It is expected that, in the absence of motion-induced MTF effects, $R_{0}$ will increase with increasing resolution to the limit of atmospheric attenuation. The observed curves validate the hypothesis, with lower resolution curves seeing a corresponding decrease in detection range. It is noted that sub-sampling is equivalent to averaging, reducing the impact of random jitter at the expense of reduced range performance. This suggests that sensors with a smaller pixel pitch, if adequately sub-sampled, can reduce the observed target jitter.

\section{EVALUATION OF MULTIPLE TRIALS}

Robustness of the methodology was tested by repeating the analysis for all relevant (head-on and collision offset) flight trials from the February dataset. The results were further compared to flight trials from the March dataset for consistency and repeatability. Results of both datasets are presented below with supporting analysis. In summary, the intra-day trials were very consistent, with variations in illumination, predominantly due to the change in sun angle, handled appropriately by the technique. Comparison between days also proved to be consistent, with both values well within their respective margins of error, suggestive of a highly robust technique.

\subsection{Intra-Day Evaluation}

Fig. 9a shows SNR curves plotted against range for multiple head-on and azimuthal-offset trials were flown on Feb 28 2012. The $R_{0}$ values for each trial are extracted as per the described methodology and enumerated in Table 2. Each row in Table 2 represents a single trial. All angular values in the table are recorded from the perspective of the host platform.

The initial column lists the collision geometry flown, as per Fig. 4. Time-stamps correspond to the start of the image acquisition. The heading value listed for each trial represents the median estimated over the trial duration. Sun azimuth values were calculated relative to the host platform from GPS data at the start of each trial. Elevation measures the sun's vertical angular offset from the horizon. All degree-valued measurements (heading, azimuth, elevation) assume a two-standard-deviation error of \pm 5.0 degrees. A mean $\overline{R_{0}}=6.3 \pm 1.7 \mathrm{~km}$ was calculated across all trials, and indicates an error much larger than the precision of a single trial. It is hypothesized that the trial-to-trial variability in $R_{0}$ can be attributed to systematic bias between trials, which may be caused by a confluence of factors such as changes in the sun angle, the trajectory geometry and windinduced target attitude variations. Note that the large systematic error in $R_{0}$ (compared to its random error) suggests that the comparison of two or more aircraft detection sensors is best performed by operating all of them concurrently. 


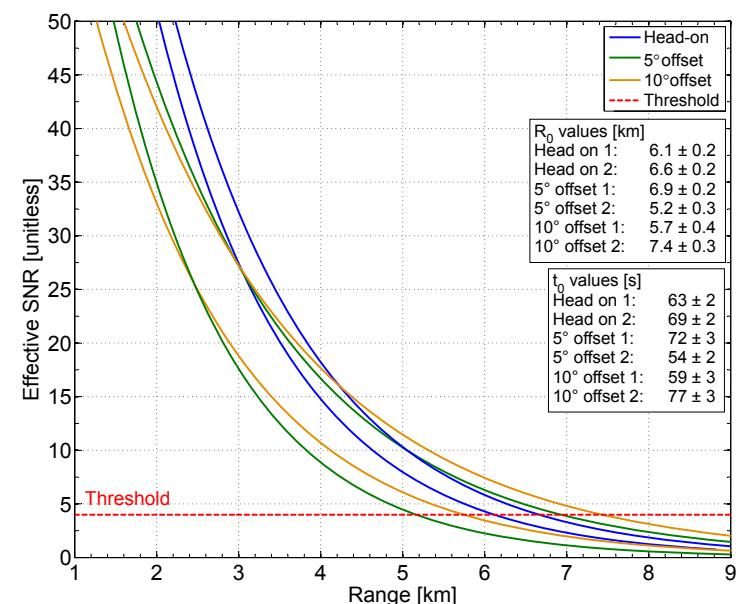

(a) Evaluation of SNR curves for multiple trials on February 28 2012. Individual $R_{0}$ and $t_{0}$ values are listed for convenience, representing a total of six trials, with details in Table2.

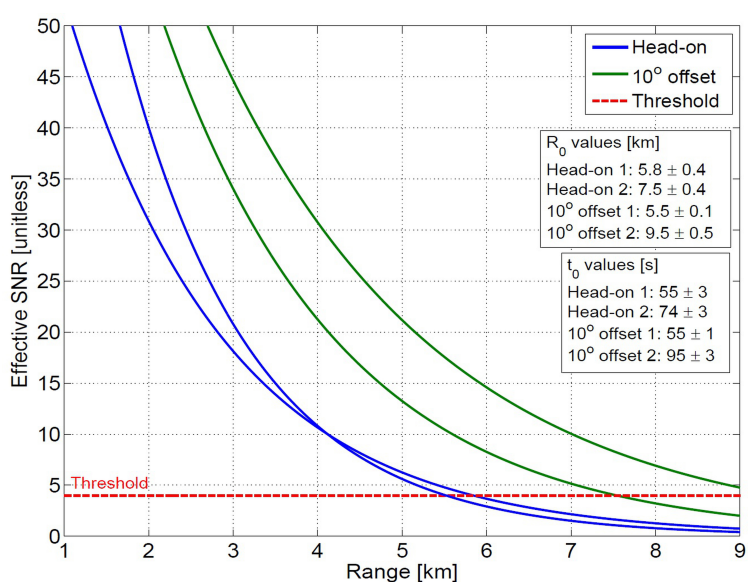

(b) Evaluation of SNR curves for multiple events from the March 2012 collection. Individual $R_{0}$ and $t_{0}$ values are listed for convenience, representing a total of four trials, with details in Table 3.

Figure 9. Results of multiple collision trajectories for February 2012 and March 2012

Table 2. Statistics on head-on and offset collision geometries for February 282012 .

\begin{tabular}{|l|c|c|c|c|c|}
\hline Type & $\begin{array}{c}\text { Time } \\
{[\mathbf{h : m}]}\end{array}$ & $\begin{array}{c}\text { Heading } \pm 2 \sigma \\
{[\mathbf{d e g}]}\end{array}$ & $\begin{array}{c}\text { Sun }(\mathbf{A z}, \mathbf{E l}) \\
{[\mathbf{d e g}, \mathbf{d e g}]}\end{array}$ & $\begin{array}{c}\mathbf{R}_{\mathbf{0}} \pm \mathbf{2} \sigma \\
{[\mathbf{k m}]}\end{array}$ & $\begin{array}{c}\mathbf{t}_{\mathbf{0}} \pm \mathbf{2} \sigma \\
{[\mathbf{s}]}\end{array}$ \\
\hline Head-on & $15: 55$ & $-25 \pm 2.5$ & $(-96,17)$ & $6.1 \pm 0.2$ & $63 \pm 2$ \\
\hline $5^{\circ}$ offset & $16: 00$ & $160 \pm 2.2$ & $(80,17)$ & $6.9 \pm 0.2$ & $69 \pm 2$ \\
\hline $10^{\circ}$ offset & $16: 04$ & $-14 \pm 1.8$ & $(-105,16)$ & $5.8 \pm 0.4$ & $72 \pm 3$ \\
\hline Head-on & $16: 09$ & $160 \pm 2.2$ & $(80,15)$ & $6.7 \pm 0.2$ & $54 \pm 2$ \\
\hline $5^{\circ}$ offset & $16: 13$ & $1.0 \pm 1.8$ & $(-119,15)$ & $5.2 \pm 0.3$ & $59 \pm 3$ \\
\hline $10^{\circ}$ offset & $16: 17$ & $170 \pm 1.5$ & $(80,14)$ & $7.4 \pm 0.3$ & $77 \pm 3$ \\
\hline Mean & - & - & - & $\mathbf{6 . 3} \pm \mathbf{1 . 7}$ & $\mathbf{6 6} \pm \mathbf{1 9}$ \\
\hline
\end{tabular}

Table 3. Statistics on head-on and offset collision geometries for March 07, 2012.

\begin{tabular}{|l|c|c|c|c|c|}
\hline Type & $\begin{array}{c}\text { Time } \\
{[\mathbf{h : m}]}\end{array}$ & $\begin{array}{c}\text { Heading } \pm 2 \sigma \\
{[\mathbf{d e g}]}\end{array}$ & $\begin{array}{c}\text { Sun }(\mathbf{A z}, \mathbf{E l}) \\
{[\mathbf{d e g}, \mathbf{d e g}]}\end{array}$ & $\begin{array}{c}\mathbf{R}_{\mathbf{0}} \pm \mathbf{2} \sigma \\
{[\mathbf{k m}]}\end{array}$ & $\begin{array}{c}\mathbf{t}_{\mathbf{0}} \pm \mathbf{2} \sigma \\
{[\mathbf{s}]}\end{array}$ \\
\hline Head-on & $9: 35$ & $-120 \pm 2.8$ & $(68,25)$ & $5.8 \pm 0.4$ & $55 \pm 3$ \\
\hline $10^{\circ}$ offset & $9: 44$ & $65 \pm 5.0$ & $(-102,28)$ & $5.5 \pm 0.4$ & $74 \pm 3$ \\
\hline Head-on & $9: 49$ & $-110 \pm 2.2$ & $(71,29)$ & $7.5 \pm 0.2$ & $53 \pm 1$ \\
\hline $10^{\circ}$ offset & $9: 55$ & $88 \pm 3.2$ & $(-107,30)$ & $9.4 \pm 0.3$ & $95 \pm 3$ \\
\hline Mean & - & - & - & $\mathbf{7 . 1} \pm \mathbf{1 . 8}$ & $\mathbf{6 9} \pm \mathbf{2 0}$ \\
\hline
\end{tabular}

\subsection{Inter-Day Validation}

The flight tests were repeated on Mar 7, 2012 at CYOW to validate the proposed technique's robustness against time-of-day effects, weather fluctuations and other ancillary factors. Flights were conducted in the morning, with twelve trajectories flown over the period of one hour. Of these, four of were head-on and azimuthal offset collision trajectories. Atmospheric conditions consisted of clear-sky visibility, in excess of 15 standard miles as per the METAR profile. Ancillary conditions (temperature, pressure, humidity, UV) were similar, if not identical, to the February collection. The noticeable exception were prevailing cross-winds, with gusts on occasion exceeding 24 knots, for the duration of the collection. Thunder-showers were predicted from noon onwards, limiting the flight test window.

Fig. 9b illustrates the SNR as a function of range, calculated as per the above-mentioned methodology. The analysis pipeline was kept identical in all respects to that applied to the February collection, with the only changes being the path indicating the location of the new imagery and the number of images collected. A 
preliminary overview indicates that the data is consistent and in accordance with the model. A constant heading proved difficult to maintain due to strong cross-winds. Frequent course corrections and side-slip were inevitable despite best efforts. Variations in the observed signal are larger as a result (Table 3) and target drop-outs are observed in some of the trials. This effect was most felt the 10 degree azimuthal offset cases, which were at the limit of the field-of-view of the instrument. Given the robustness of the methodology, $R_{0}$ can still be estimated for those trials, albeit with a corresponding increase in error (Table 3).

The March dataset is processed by repeating the proposed methodology, and is observed to have a mean $\overline{R_{0}}$ value of $\overline{R_{0}}=7.1 \pm 1.8 \mathrm{~km}$. Both the mean and the standard deviation are comparable to the expected value acquired from the February dataset of $\overline{R_{0}}=6.3 \pm 1.7 \mathrm{~km}$, affirming the reproducibility of the technique. The cost of developing each unique system and conducting flight tests is prohibitive and should not be underestimated. The methodology described herein will be utilized to validate additional sensing modalities as industrial capability in this problem space matures.

\subsection{Inter-Instrument Validation}

Finding a common basis for comparison is a challenging task when performing flight tests in real world conditions. For example, local atmospheric conditions vary both spatially (e.g. clouds and precipitation) and temporally. This is often true despite the aggregate meteorological assessment of a clear sky. As well, sun angle is continuously changing throughout the day. Because of these local variations, any two instruments can only be compared on an identical basis if they are flown simultaneously on the same aircraft.

To that end, the NRC-developed Cerberus, a three node array of high-quality, monochrome, machine vision cameras, was flown in a dual sensor installation with DragonflEYE during the March 2012 flights. Both Cerberus and DragonflEYE are EO arrays, with Cerberus based on CCD technology and DragonflEYE on CMOS technology. Salient parameters for Cerberus are detailed in Table 4. In Fig. 3a Cerberus is the black instrument located mid-way on the port side, while DragonflEYE is the orange instrument mounted underneath the nose of the 205. Two head-on collision trajectories captured by the forward-facing camera were chosen for comparison. These image sequences were recorded simultaneously with the head-on cases from DragonflEYE in Fig. 9b and Table 3 , and represent an ideal basis for comparison given that the environmental transfer functions $\left(M_{A}, M_{V}\right)$ were identical for both sensors. As per the analysis in Section 6.4, the $R_{0}$ value will scale with angular resolution. Note that the angular resolution for the forward-facing camera in Cerberus is $0.15 \mathrm{mrad}$, which is a factor of 1.5 lower than the $0.1 \mathrm{mrad}$ resolution of DragonflEYE. Therefore, the $R_{0}$ value should be diminished by a factor of 1.5 as well. Table 5 depicts the results of this comparison, with the predicted and measured values compared. It can be observed that the predicted value correlates strongly with the measured $R_{0}$ values for Cerberus, confirming $R_{0}$ as a robust, reliable maximum sensing range for EO sensors that is dominated by atmospheric effects.

\section{CONCLUSION}

The methodology for evaluating the range performance of an EO instrument in a collision detection application is presented in this work. A trajectory-scale figure of merit, "range at first detection" $\left(R_{0}\right)$, based on the variation of an empirical SNR of the target aircraft, was presented as a measure of sensor performance. The methodology was evaluated by conducting flight tests with a prototype EO camera array, DragonflEYE, mounted onboard a surrogate UAS. Actual collision geometries were flown against a manned intruder over two days. Although the technique was used with EO cameras, it can be extended to other modalities with minimal effort. A single airborne estimate of $R_{0}=6.7 \pm 0.2 \mathrm{~km}$ for a head-on collision under typical visual meteorological conditions proved to be more precise than the typical pilot measurement, $R_{v}$, under identical conditions $\left(R_{v}=4.8 \pm 2.2 \mathrm{~km}\right)$. Comparing $R_{0}$ values over multiple trials $\left(\bar{R}_{0}=6.3 \pm 1.7 \mathrm{~km}\right)$ reveals larger variations, which can be attributed primarily to changing scene conditions. Such variability is expected, given the difficulty in obtaining identical scene conditions for multiple trials. Sensitivity analysis confirmed that increasing resolution will increase $R_{0}$ to the limit of atmospheric extinction. Validation was performed by repeating the experiment on a different day under similar visual conditions. Similarly, robustness was confirmed by comparing the results to another multicamera array, Cerberus, flown simultaneously during the March 2012 test flights. In both cases, the predicted behaviour correlated strongly with the measurement. 
Table 4. Cerberus parameters for the March 2012 dataset

\begin{tabular}{|l|c|c|}
\hline Parameter & Value & Units \\
\hline Camera count & 2 & - \\
Azimuth configuration & $\left(0^{\circ}, 45^{\circ}\right)$ & $\mathrm{deg}$ \\
Total FOV & $75^{\circ} \times\left(18^{\circ}, 50^{\circ}\right)$ & $\mathrm{deg}$ \\
Focal length $(f)$ & $0^{\circ}=23,45^{\circ}=8$ & $\mathrm{~mm}$ \\
Focal ratio $(\mathrm{F} \#)$ & 2.8 & - \\
Angular resolution & $0^{\circ}=0.15,45^{\circ}=0.43$ & $\mathrm{mrad} / \mathrm{pixel}$ \\
Pixel count & $2448 \times 2050 \times 2$ & $\mathrm{pixel}$ \\
\hline
\end{tabular}

Table 5. Comparison between Cerberus and DragonflEYE on head-on collision geometries for March 072012 . Predicted ranges were computed by scaling the resolution as indicated in Section 6.4

\begin{tabular}{|l|c|c|c|c|c|}
\hline Type & $\begin{array}{c}\text { Time } \\
{[\mathbf{h}: \mathbf{m}]}\end{array}$ & $\begin{array}{c}\text { Heading } \pm 2 \sigma \\
{[\mathbf{d e g}]}\end{array}$ & $\begin{array}{c}\text { DragonflEYE } \\
\mathbf{R}_{\mathbf{0}} \pm \mathbf{2} \sigma[\mathbf{k m}]\end{array}$ & $\begin{array}{c}\text { Cerberus Predicted } \\
\mathbf{R}_{\mathbf{0}}[\mathbf{k m}]\end{array}$ & $\begin{array}{c}\text { Cerberus Measured } \\
\mathbf{R}_{\mathbf{0}} \pm \mathbf{2} \sigma[\mathbf{k m}]\end{array}$ \\
\hline Head-on & $9: 35$ & $-120 \pm 2.8$ & $5.8 \pm 0.4$ & 3.9 & $3.9 \pm 0.1$ \\
\hline Head-on & $9: 49$ & $-110 \pm 2.2$ & $7.5 \pm 0.2$ & 5.0 & $4.9 \pm 0.1$ \\
\hline Mean & - & - & $\mathbf{5 . 7} \pm \mathbf{0 . 3}$ & $\mathbf{4 . 5}$ & $\mathbf{4 . 4} \pm \mathbf{0 . 7}$ \\
\hline
\end{tabular}

The current regulatory climate necessitates the use of manned aircraft to conduct airborne collision geometries crucial towards generating suitable data. In the future, as requirements are more clearly defined, similar testing is expected to be conducted between unmanned aircraft and manned intruders. To that end, arrays of EO sensors are readily miniaturized and represent the most straightforward path towards integration on light UAS platforms.

\section{Acknowledgments}

The authors thank the pilots Robert Erdos and Stephan Carignan (1964-2014) for their expert flying, Dr. Gregory Craig for his assistance during flight tests, students Paris Ang, Gary Zeng and Jeremy Chan-Hao Wang for their diligence in target selection, and students Hou In (Stanley) Lio, Kyle Watters and Paris Ang for their programming efforts. The authors acknowledge Public Works Canada and the Ontario Centres of Excellence for their financial support.

\section{References}

Andrews, J. (1991). Unalerted Air-to-Air Visual Acquisition - ATC 152. Technical report, Lincoln Laboratories, MIT, Lexington, MA.

Born, M. and Wolf, E. (1999). Principles of Optics: Electromagnetic Theory of Propagation, Interference and Diffraction of Light. Cambridge University Press, 7th edition.

Brady, D. J., Gehm, M. E., Stack, R. A., Marks, D. L., Kittle, D., Golish, D., Vera, E. M., and Feller, S. (2012). Multiscale gigapixel photography. Nature, 486:386-389.

Brown, D. C. (1971). Close-range camera calibration. Photogrammetric Engineering and Remote Sensing, $37(8): 855-866$.

Dey, D., Geyer, C., Singh, S., and Digioia, M. (2009). Passive, long-range detection of aircraft: Towards a field deployable Sense and Avoid System. In Proc. Conference on Field and Service Robotics, pages 1-10.

Dey, D., Geyer, C., Singh, S., and Digioia, M. (2011). A Cascaded Method to Detect Aircraft in Video Imagery. International Journal of Robotics Research, 30(12):1527-1540.

Ebdon, D. and Regan, J. (2004). Sense-and-avoid requirement for Remotely Operated Aircraft (ROA). Technical report, Langley Air Force Base, USAF.

Ellis, K. and Gubbels, A. W. (2005). The National Research Council of Canada's Surrogate UAV Facility. In Proc. UVS Canada Conference. 
Etkin, B. and Reid, L. D. (1995). Dynamics of Flight: Stability and Control. John Wiley and Sons, 3rd edition.

Fasano, G., Accardo, D., Tirri, A. E., Moccia, A., and Lellis, E. D. (2014). Morphological Filtering and Target Tracking for Vision-based UAS Sense and Avoid. In Proc. International Conference on Unmanned Aircraft Systems, pages 430-440.

Fasano, G., Moccia, A., Accardo, D., and Rispoli, A. (2008). Development and test of an integrated sensor system for autonomous collision avoidance. In Proc. International Congress of the Aeronautical Sciences.

Federal Aviation Administration (FAA) (1983). Advisory Circular 90-48C: Pilots' role in collision avoidance. Technical report, US DOT, FAA.

Forlenza, L., Carton, P., Accardo, D., Fasano, G., and Moccia, A. (2012). Real Time Corner Detection for Miniaturized Electro-Optical Sensors Onboard Small Unmanned Aerial Systems. Sensors (Basel, Switzerland), 12(1):863-77.

Griffith, J. D. and Kuchar, J. K. (2008). Electro-Optical System Analysis for Sense and Avoid. In AIAA Conference on Guidance, Navigation and Control, pages 18-21.

Grilley, D. E. (2005). Resolution requirements for passive sense-and-avoid. Technical report, Alion Science and Technology.

Holst, G. C. (2003). Electro-optical Imaging System Performance. SPIE Press, 3rd edition.

Janesick, J. R. (2001). Scientific Charge-coupled Devices. SPIE Press, 1st edition.

Keillor, J., Ellis, K., Craig, G., Rozovski, D., and Erdos, R. (2011). Studying collision avoidance by nearly colliding: A flight test evaluation. In Proc. Human Factors and Ergonomics Annual Meeting, pages 41-45.

Kim, I. S., Choi, H. S., Yi, K. M., Choi, J. Y., and Kong, S. G. (2010). Intelligent visual surveillance - a survey. International Journal of Control, Automation and Systems, 8(5):926-939.

Kopeika, N. S. (1998). A System Engineering Approach to Imaging. SPIE Press, 1st edition.

Lai, J., Ford, J. J., Mejias, L., and O'Shea, P. (2013). Characterization of Sky-region Morphological-temporal Airborne Collision Detection. Journal of Field Robotics, 30(2):171-193.

Leach, B. W., Dillon, J., and Rahbari, R. (2003). Operational experience with optimal integration of low-cost inertial sensors and gps for flight test requirements. Canadian Aeronautics and Space Journal, 49(2):41-54.

Mahajan, V. N. (1994). Zernike circle polynomials and optical aberrations of systems with circular pupils. Applied Optics, 33(34):8121-8121.

McCalmont, J., Utt, J., Deschenes, M., Taylor, M., Sanderson, R., Montgomery, J., Johnson, R., and McDermott, D. (2007). Sense and avoid technology for unmanned aircraft systems. In Proc. SPIE Conference on Automatic Target Recognition XVII, volume 6566 .

McGuire, J. (2010). Manufacturable mobile phone optics: Higher order aspheres are not always better. In Proc. OSA International Conference on Optical Design.

Mendelson, J. (2014). Innovations in Unmanned Vehicles - Land, Air, Sea (Technical Insights). Technical Report D56B-01, Frost and Sullivan.

Minwalla, C. (2013). DragonflEYE: A Passive Approach to Aerial Collision Sensing. PhD thesis, York University.

Minwalla, C., Thomas, P., Ellis, K., Hornsey, R., and Jennings, S. (2012). Flight test evaluation of a prototype optical instrument for airborne sense-and-avoid applications. In Proc. SPIE Conference on Defense, Security and Sensing - Unmanned Systems Technology, pages 83870R-1-14. 
Overington, I. (1976). Vision and Acquisition: Fundamentals of human visual performance, environmental influences and applications in instrumentational optics. Pentech Press Limited, 1st edition.

Raty, T. D. (2010). Survey on contemporary remote surveillance systems for public safety. IEEE Transactions on Systems, Man, and Cybernetics, Part C: Applications and Reviews, PP(99):1-23.

Shen, E. (2012). Optimization of Resource Allocation using Self-Organization in an Embedded Distributed Smart Camera Network. PhD thesis, York University. 